\title{
Plasma Asprosin, CCDC80 and ANGPTL4 Levels Are Associated With Metabolic and Cardiovascular Risk in Patients With Inflammatory Bowel Disease
}

\author{
Hao-Hua WANG ${ }^{1}$, Wan-Ying LUO ${ }^{1}$, Min LIN ${ }^{1}$, Xiao-Jing LI ${ }^{1}$, Guang-Da XIANG ${ }^{2}$, Silvia D \\ TRIGANTI ${ }^{3}$
}

${ }^{1}$ Department of Endocrinology, Tungwah Hospital of Sun Yat-Sen University, Dongguan, China, ${ }^{2}$ Department of Endocrinology, Wuhan General Hospital of Guangzhou Command, Wuhan, China, ${ }^{3}$ University of Maryland, Baltimore, USA.

Received July 22, 2020

Accepted February 12, 2021

Epub Ahead of Print March 8, 2021

\section{Summary}

Asprosin, coiled-coil domain-containing 80(CCDC80) and angiopoietin-like 4(ANGPTL4) are newly discovered adipocytokine that affects glucose tolerance, insulin resistance and cardiovascular diseases. The goal of this study was to investigate if a relationship exists among asprosin, CCDC80 and ANGPTL4 and inflammatory bowel disease (IBD). Fifty subjects with newly diagnosed IBD and fifty healthy individuals were enrolled. Patients were treated with standard therapies for 3 months. Plasma asprosin, CCDC80 and ANGPTL4 levels were measured with enzyme-linked immunosorbent assay. High resolution ultrasound was used to measure brachial artery diameter at rest, after reactive hyperemia (flow-mediated dilation, FMD) and after sublingual glyceryltrinitrate. Compare with healthy individuals, plasma CCDC80, erythrocyte sedimentation rate (ESR), C-reactive protein (CRP) levels and homeostasis model assessment of insulin resistance (HOMA-IR) were significantly higher ( $p<0.05$, respectively), whereas plasma asprosin, ANGPTL4 levels and FMD were significantly lower in both UC and CD patients $(p<0.05)$. Plasma CCDC80 levels were significantly higher in patients with CD $(p<0.05)$, while plasma asprosin and ANGPTL4 levels were lower $(\mathrm{pP}<0.05)$ as compared with those in patients with UC. Standard therapies increased plasma asprosin, ANGPTL4 levels and FMD in both UC and CD $(p<0.05)$, UC and CD patientswhile decreased plasma CCDC80, ESR, CRP levels and HOMA-IR $(p<0.05)$. The changes in HOMA-IR and FMD were correlated with the changes in plasma asprosin, CCDC80 and ANGPTL4 levels over the study period $(p<0.05)$. Plasma asprosin, CCDC80 and ANGPTL4 levels may be applied as a significant marker for early stage of insulin resistance and atherosclerosis in IBD, especially of $C D$.

\section{Key words}

Inflammatory bowel disease - Endothelial function - Insulin resistance $\bullet$ Adipocytokine

\section{Corresponding author}

Wang Hao-hua, Department of Endocrinology, Tungwah Hospital of Sun Yat-Sen University, Dongguan 523110, Guangdong Province, China. E-mail: hello_vanwang@sina.com

\section{Introduction}

Both ulcerative colitis (UC) and Crohn's disease (CD) are forms of inflammatory bowel disease (IBD), which is characterized by chronic, progressive inflammation of the gastrointestinal tract (Korkmaz et al. 2014, Ng et al. 2018). Epidemiological studies have reported increased risk of atherosclerosis in CD and UC, and clinical studies have evidenced accelerated insulin resistance in IBD (Korkmaz et al. 2014, $\mathrm{Ng}$ et al. 2018). Endothelial dysfunction is the early development of atherosclerosis and consequently cardiovascular complications (Celermajer et al. 1992). Clinical studies have showed that the impairment of FMD exists in IBD (Cibor et al. 2016, Jehlička et al. 2014, Morhardt et al. 2019). The adipose tissue of the human body is not only an organ that stores energy but also an endocrine tissue 
that is active in metabolism. It secretes hormones and a variety of other biologically active substances and participates in maintaining many physiological functions of the body. The adipokines asprosin, coiled-coil domaincontaining $80(\mathrm{CCDC} 80)$ and angiopoietin-like 4(ANGPTL4) are involved in the progression of insulin resistance and cardiovascular diseases (McCulloch et al. 2020, Min et al. 2019, O Osorio-Conles et al. 2017, Shota et al. 2016). However, to date; no data are available on the effect of asprosin, CCDC80 and ANGPTL4 in insulin resistance and cardiovascular diseases IBD. Therefore, we hypothesized that plasma asprosin, CCDC80 and ANGPTL4 levels are associated with early stage of insulin resistance and atherosclerosis in IBD.

\section{Material and Methods}

\section{Subjects}

From Nov 2017 to July 2020, 50 newly diagnosed IBD (23 with ulcerative colitis (UC) 25 with Crohn's disease (CD) and 2 with unclassified IBD) and 50 healthy volunteers (control-a), referred to our hospital for healthy examination, and between 15 to 50 years of age were studied. Exclusion criteria were according to previous study by us (Hao-hua et al. 2018). All participants who presented a history of congestive heart failure, myocardial infarction or angina were excluded from this study. Besides, Those people with obese (BMI > $30 \mathrm{~kg} / \mathrm{m} 2$ ), malignant neoplasms, hypertension, renal or liver diseases were also excluded from our study.

Patients were administered with standard therapies (Ng et al. 2018) for 3 months. To decide which treatment will be successful for a given patient, disease severity, location, phenotype, effect of the disease on growth and development, and the psychosocial status of the patient should all be considered. The approach uses drugs such as aminosalicylates, antibiotics, or enteral therapy and escalates to immunomodulators, biologics.

Besides, 30 subjects with bacterial or viral bowel inflammation (mean age $33.9 \pm 2.9$ years) were studied as control-b.

The disease activity was determined by the $\mathrm{CD}$ activity index in $\mathrm{CD}$ and the Mayo score in UC. CD activity index ranges from 0 to 600 . Total Mayo score ranges from 0 to 12 .

The duration of unresolved gastrointestinal symptoms and predictors of timely specialist review before the diagnosis of both $\mathrm{CD}$ and $\mathrm{UC}$ is recorded in our study.

This study has been reviewed by an appropriate ethics committee and has been conducted in accordance with the ethical standards laid down by the1964 Declaration of Helsinki. Written informed consent was obtained from all subjects.

\section{Laboratory methods}

The procedure was described previously (Hao-hua et al. 2018). Venous blood samples were drawn after a 12 to $14 \mathrm{~h}$ overnight fast. Plasma samples were analysed for asprosin, CCDC80 and ANGPTL4 content by ELISA (Shino-Test, IBL) according to the manufacturer's instructions, with samples diluted 1 to 2 in assay buffer and a standard curve ranging from $80 \mathrm{ng} / \mathrm{ml}$ to $300 \mathrm{pg} / \mathrm{ml}$. Plasma samples were diluted 1 to 3 and measured in duplicate, and the results were averaged.

Other parameters, including total cholesterol (TC), triglycerides (TG), high-density lipoprotein (HDL), low-density lipoprotein (LDL), HbA1C, uric acid and urine albumin were enzymatically determined in the laboratory of the authors' hospital. The fasting blood glucose (FBG) was analyzed by a glucose oxidase method. Levels of ultrasensitive C-reactive protein (CRP) were analyzed by immunoturbidimetric analysis. Erythrocyte sedimentation rate (ESR) and creatinine were determined using an automated analyzer (Sysmex XE5000, Japan). Insulin resistance was estimated by the homeostasis model assessment of insulin resistance (HOMA-IR) defined as fasting glucose $(\mathrm{mmol} / \mathrm{L}) \times$ fasting insulin $(\mathrm{mu} / \mathrm{l}) / 22.5$.

\section{Ultrasound study of the brachial artery}

The vascular studies of the brachial artery were performed non-invasively, as described by us previously (Hao-hua et al. 2018). High resolution ultrasound was used to measure changes in arterial diameter in response to reactive hyperemia (with increased flow producing an endothelium-dependent stimulus to vasodilation; FMD) and to glyceryltrinitrate (GTN-induced endotheliumin dependent arterial dilation) (128XP/10 with a 7. 0-MHz linear array transducer; Acuson, Mountain View, CA, USA). The intra- and inter-observer variability in our laboratory for repeated measurements of artery diameter is $0.09 \pm 0.10 \mathrm{~mm}$ and $0.08 \pm 0.13 \mathrm{~mm}$, respectively. The $\mathrm{CV}$ for FMD measurements over time is $7.8-9.2 \%$; the power of the study to justify the selected 
sample size is $83.7 \%$, and the resolving power of the method tested on phantom arteries is $8.6 \mu \mathrm{m}$.

\section{Statistical methods}

The differences for numerical variables among different groups were analyzed by the ANOVA. Chi-square was performed to compare categorical variables and nominal variables. Univariate analysis of the effects of each potential risk factor on HOMA-IR and FMD was performed with linear regression for continuous variables (vessel size, blood flow, GTNinduced endothelium-independent arterial dilation, asprosin, CCDC80 and ANGPTL4, LDL-C, HDL-C, TC, TG, CRP, DBG, SBG, FBG, age, ESR, persistence of symptom, HbA1C, uric acid, urine albumin and BMI) and with one-way ANOVA for categorical variables (sex and smokers). Linear regression analyses were used to assess the relation between the therapy-induced changes in HOMA-IR, FMD and other variables.

Table 1. Clinical and biochemical characteristics in different groups. $(\chi \pm s)$

\begin{tabular}{|c|c|c|c|c|}
\hline & \multirow{2}{*}{ Control-a } & \multirow{2}{*}{ Control-b } & \multicolumn{2}{|c|}{ IBD before treatment } \\
\hline & & & $\mathbf{U C}$ & CD \\
\hline Number of subjects & 50 & 30 & 23 & 25 \\
\hline Age (years) & $33 \pm 11.5$ & $33.9 \pm 2.9$ & $34.3 \pm 12.3$ & $36.7 \pm 10.8$ \\
\hline Smoker [n (\%)] & $3(6 \%)$ & $3(10 \%)$ & $2(8.6 \%)$ & $3(12 \%)$ \\
\hline $\operatorname{Sex}(M / F)$ & $20 / 30$ & $17 / 13$ & $11 / 12$ & $12 / 13$ \\
\hline$S B P(m m H g)$ & $118.3 \pm 11.8$ & $101.3 \pm 12.3$ & $120.8 \pm 14.5$ & $125.3 \pm 11.8$ \\
\hline$D B P(m m H g)$ & $72.2 \pm 13.2$ & $73.1 \pm 10.5$ & $70.7 \pm 12.6$ & $77.2 \pm 15.1$ \\
\hline$B M I(\mathrm{~kg} / \mathrm{m} 2)$ & $23.5 \pm 7.1$ & $22.12 \pm 5.1$ & $25.9 \pm 2.1$ & $24.5 \pm 3.2$ \\
\hline FPG $(\mathrm{mmol} / \mathrm{l})$ & $4.31 \pm 2.34$ & $4.51 \pm 1.66$ & $5.09 \pm 1.67$ & $5.26 \pm 1.23$ \\
\hline TC $(\mathrm{mmol} / \mathrm{l})$ & $4.08 \pm 1.55$ & $3.95 \pm 1.35$ & $4.37 \pm 1.56$ & $4.29 \pm 1.43$ \\
\hline$L D L-C(\mathrm{mmol} / \mathrm{l})$ & $2.32 \pm 0.62$ & $2.52 \pm 0.65$ & $2.67 \pm 0.55$ & $2.45 \pm 1.09$ \\
\hline$H D L-C(\mathrm{mmol} / \mathrm{l})$ & $1.78 \pm 0.31$ & $1.99 \pm 0.77$ & $1.25 \pm 0.89$ & $1.11 \pm 0.56$ \\
\hline$T G(\mathrm{mmol} / \mathrm{l})$ & $1.52 \pm 0.62$ & $1.81 \pm 0.86$ & $1.75 \pm 0.86$ & $1.91 \pm 1.09$ \\
\hline$H b A 1 C(\%)$ & $4.13 \pm 1.23$ & $4.34 \pm 1.56$ & $4.75 \pm 1.08$ & $4.62 \pm 1.05$ \\
\hline Uric acid (umol/l) & $313.6 \pm 27.9$ & $333.8 \pm 33.6$ & $309.1 \pm 27.9$ & $318.5 \pm 25.1$ \\
\hline Urine Albumin $(\mathrm{mg} / \mathrm{l})$ & $12.9 \pm 6.4$ & $15.6 \pm 8.7$ & $10.7 \pm 7.9$ & $13.6 \pm 8.5$ \\
\hline$H O M A-I R$ & $2.25 \pm 1.45$ & $2.05 \pm 1.35$ & $3.93 \pm 1.65 * 0$ & $4.17 \pm 1.66 * 0$ \\
\hline Asprosin $(n g / m l)$ & $28.38 \pm 7.11$ & $27.11 \pm 6.45$ & $17.89 \pm 7.22 * q$ & $12.77 \pm 9.09 *$ 中\# \\
\hline CCDC80 $(\mathrm{ng} / \mathrm{ml})$ & $1.15 \pm 0.33$ & $1.35 \pm 0.78$ & $1.78 \pm 1.68^{*}+$ & $2.33 \pm 1.86^{*}+\#$ \\
\hline ANGPTL4 $(\mathrm{pg} / \mathrm{ml})$ & $42.13 \pm 5.12$ & $45.35 \pm 6.12$ & $25.11 \pm 12.21 * q$ & $20.56 \pm 13.77 *$ 中\# \\
\hline$C R P(m g / l)$ & $1.08 \pm 0.89$ & $2.15 \pm 1.07$ & $3.97 \pm 0.67 * *$ & $4.06 \pm 0.86 * * \circ$ \\
\hline $\operatorname{ESR}(\mathrm{mm} / \mathrm{h})$ & $3.3 \pm 1.32$ & $5.12 \pm 1.67$ & $17.11 \pm 2.66^{* *}$ & $14.88 \pm 3.79 * * q$ \\
\hline Disease activity & - & - & $6.86 \pm 2.67$ & $278.23 \pm 35.23$ \\
\hline Duration of symptoms (year) & - & - & $3.21 \pm 1.52$ & $4.12 \pm 2.12$ \\
\hline Baseline flow ( $\mathrm{ml} / \mathrm{min})$ & $80.55 \pm 36.23$ & $83.15 \pm 31.55$ & $81.23 \pm 25.77$ & $86.33 \pm 26.67$ \\
\hline GTN-induced dilation (\%) & $23.12 \pm 5.01$ & $25.55 \pm 3.77$ & $26.37 \pm 6.55$ & $22.56 \pm 3.11$ \\
\hline$F M D(\%)$ & $5.65 \pm 1.01$ & $5.71 \pm 1.67$ & $3.51 \pm 2.23 * 0$ & $3.22 \pm 1.57 * * q$ \\
\hline Baseline vessel (mm) & $4.03 \pm 1.12$ & $4.15 \pm 2.08$ & $3.69 \pm 1.33$ & $3.92 \pm 1.28$ \\
\hline
\end{tabular}

${ }^{*} p<0.05, * * p<0.01$, compared with control-a, $q \mathrm{p}<0.05$, + $+\mathrm{p}<0.01$, compared with control-b, \#p $<0.05, \#$ \# $<0.01$, UC vs. CD. ANGPTL4, angiopoietin-like 4; CCDC80, coiled-coil domain-containing 80; CRP, C-reactive protein; ESR, erythrocyte sedimentation rate; FPG, fasting glucose; FMD, flow-mediated dilation; HOMA-IR, homeostasis model assessment of insulin resistance; HDL-C, highdensity lipoprotein cholesterol; LDL-C, low-density lipoprotein cholesterol; TC, total cholesterol; TG, triglyceride; 
Table 2. Clinical and biochemical characteristics in UC and CD $(\chi \pm s)$

\begin{tabular}{|c|c|c|c|c|}
\hline & \multicolumn{2}{|c|}{$\mathbf{U C}$} & \multicolumn{2}{|c|}{ CD } \\
\hline & Before treatment & After treatment & Before treatment & After treatment \\
\hline Number of subjects & 23 & 23 & 25 & 25 \\
\hline$S B P(m m H g)$ & $120.8 \pm 14.5$ & $121.5 \pm 6.7$ & $125.3 \pm 11.8$ & $126.5 \pm 6.1$ \\
\hline$D B P(m m ~ H g)$ & $70.7 \pm 12.6$ & $76.22 \pm 12.32$ & $77.2 \pm 15.1$ & $79.12 \pm 12.79$ \\
\hline$B M I\left(\mathrm{~kg} / \mathrm{m}^{2}\right)$ & $25.9 \pm 2.1$ & $25.11 \pm 1.23$ & $24.5 \pm 3.2$ & $24.23 \pm 2.11$ \\
\hline FPG $(\mathrm{mmol} / \mathrm{l})$ & $5.09 \pm 1.67$ & $5.11 \pm 1.87$ & $5.26 \pm 1.23$ & $5.20 \pm 1.83$ \\
\hline$T C(\mathrm{mmol} / \mathrm{l})$ & $4.37 \pm 1.56$ & $4.17 \pm 1.23$ & $4.29 \pm 1.43$ & $4.09 \pm 1.89$ \\
\hline$L D L-C(\mathrm{mmol} / \mathrm{l})$ & $2.67 \pm 0.55$ & $2.12 \pm 1.45$ & $2.45 \pm 1.09$ & $2.37 \pm 1.68$ \\
\hline$H D L-C(\mathrm{mmol} / \mathrm{l})$ & $1.25 \pm 0.89$ & $1.35 \pm 1.08$ & $1.11 \pm 0.56$ & $1.22 \pm 0.79$ \\
\hline$T G(\mathrm{mmol} / \mathrm{l})$ & $1.75 \pm 0.86$ & $1.78 \pm 1.09$ & $1.91 \pm 1.09$ & $1.41 \pm 1.35$ \\
\hline$H b A 1 C(\%)$ & $4.75 \pm 1.08$ & $4.51 \pm 1.68$ & $4.62 \pm 1.05$ & $4.52 \pm 1.35$ \\
\hline Uric acid (umol/l) & $309.1 \pm 27.9$ & $311.1 \pm 28.1$ & $318.5 \pm 25.1$ & $329.5 \pm 23.1$ \\
\hline Urine Albumin (mg/l) & $10.7 \pm 7.9$ & $11.09 \pm 10.2$ & $13.6 \pm 8.5$ & $12.56 \pm 5.77$ \\
\hline$H O M A-I R$ & $3.93 \pm 1.65$ & $2.56 \pm 1.98^{*}$ & $4.17 \pm 1.66$ & $3.09 \pm 1.56^{*}$ \\
\hline Asprosin $(n g / m l)$ & $17.89 \pm 7.22$ & $23.21 \pm 15.67^{*}$ & $12.77 \pm 9.09$ & $16.12 \pm 11.77^{*}$ \\
\hline CCDC80 (ng/ml) & $1.78 \pm 1.68$ & $1.26 \pm 0.68^{*}$ & $2.33 \pm 1.86$ & $1.65 \pm 0.71^{*}$ \\
\hline ANGPTL4 $(\mathrm{pg} / \mathrm{ml})$ & $25.11 \pm 12.21$ & $33.45 \pm 13.12 *$ & $20.56 \pm 13.77$ & $38.12 \pm 12.78^{*}$ \\
\hline$C R P(m g / l)$ & $3.97 \pm 0.67$ & $2.16 \pm 0.7 * *$ & $4.06 \pm 0.86$ & $1.98 \pm 0.56^{* *}$ \\
\hline $\operatorname{ESR}(\mathrm{mm} / \mathrm{h})$ & $17.11 \pm 2.66$ & $8.25 \pm 1.28 * *$ & $14.88 \pm 3.79$ & $6.07 \pm 1.67 * *$ \\
\hline Disease activity & $6.86 \pm 2.67$ & $2.97 \pm 1.89 * *$ & $278.23 \pm 35.23$ & $157.12 \pm 25.90 * *$ \\
\hline Baseline flow ( $\mathrm{ml} / \mathrm{min})$ & $81.23 \pm 25.77$ & $80.23 \pm 23.89$ & $86.33 \pm 26.67$ & $85.35 \pm 17.78$ \\
\hline GTN-induced dilation (\%) & $26.37 \pm 6.55$ & $25.25 \pm 7.26$ & $22.56 \pm 3.11$ & $20.68 \pm 5.89$ \\
\hline$F M D(\%)$ & $3.51 \pm 2.23$ & $4.25 \pm 1.56^{*}$ & $3.22 \pm 1.57$ & $4.09 \pm 1.23^{* *}$ \\
\hline Baseline vessel (mm) & $3.69 \pm 1.33$ & $3.83 \pm 1.55$ & $3.92 \pm 1.28$ & $3.80 \pm 1.65$ \\
\hline
\end{tabular}

${ }^{*} \mathrm{p}<0.05, * * \mathrm{p}<0.01$, compared with patients before treatment; ANGPTL4, angiopoietin-like 4; CCDC80, coiled-coil domain-containing 80; CRP, C-reactive protein; ESR, erythrocyte sedimentation rate; FPG, fasting glucose; FMD, flow-mediated dilation; HOMA-IR, homeostasis model assessment of insulin resistance; HDL-C, high-density lipoprotein cholesterol; LDL-C, low-density lipoprotein cholesterol; TC, total cholesterol; TG, triglyceride;

\section{Results}

Table 1 and Table 2 show the clinical and biochemical characteristics of the subjects included in the study. There is no difference between control-a and control-b in all characteristics. In our study, the CD was most frequent $(95 \%)$ with predilection for terminal ileum and right hemicolon. The location of sigmoid and rectum make up $93 \%$ of those diagnosed with UC. Given by few patients with unclassified IBD was recruited in our study, we focused on the analysis of CD and UC.

The CD or UC presented higher CCDC80 and HOMA-IR levels compared with the healthy subjects as well as lower levels of asprosin, ANGPTL4 and FMD ( $p<0.05$, respectively). Moreover, the asprosin and ANGPTL4 in CD were were significantly lower than those in UC $(\mathrm{p}<0.05)$. Compared with UC, the CCDC80 in $C D$ was significantly higher $(p<0.05)$. Other parameters, including CRP and ESR levels in both CD and UC were significantly higher than those in the healthy group $(p<0.05$, respectively). There were no significant differences in other parameters i.e. CRP, ESR, persistence of symptom, and FMD between CD and UC $(\mathrm{p}>0.05)$.

To determine any differences in serum cytokines levels and FMD from IBD and control-b, we measured the levels of asprosin, CCDC80, ANGPTL4, HOMA-IR and FMD. CD or UC presented with significantly higher ESR, CRP, CCDC80 levels and HOMA-IR. In addition, the levels of asprosin, ANGTPTL4 and FMD were lower in CD or UC than those in control-b. The asprosin $(r=0.17)$, CCDC80 
$(\mathrm{r}=-1.75), \quad$ ANGPTL4 $\quad(\mathrm{r}=0.15), \quad$ disease activity $(r=-0.32)$, CRP $(r=-0.59)$, and ESR $(r=-0.63)$ in both $\mathrm{CD}$ and UC were significantly correlated with FMD by univariate analysis (Fig. 1, Fig. 2, and Fig. 3).

Standard treatment significantly increased the levels of asprosin, ANGPTL4 and FMD and decreased the levels of CCDC80, CRP, ESR, HOMA-IR and disease activity in both $\mathrm{CD}$ and UC $(p<0.05$, respectively).

Linear correlation coefficients were used to analyze the relationship between changes in HOMA-IR, FMD and changes in other indices in both $\mathrm{CD}$ and $\mathrm{UC}$ after treatment. The results showed that changes in HOMA-IR and FMD were all correlated with changes in asprosin, CCDC80 and ANGPTL4 $(\mathrm{p}<0.05$, respectively).
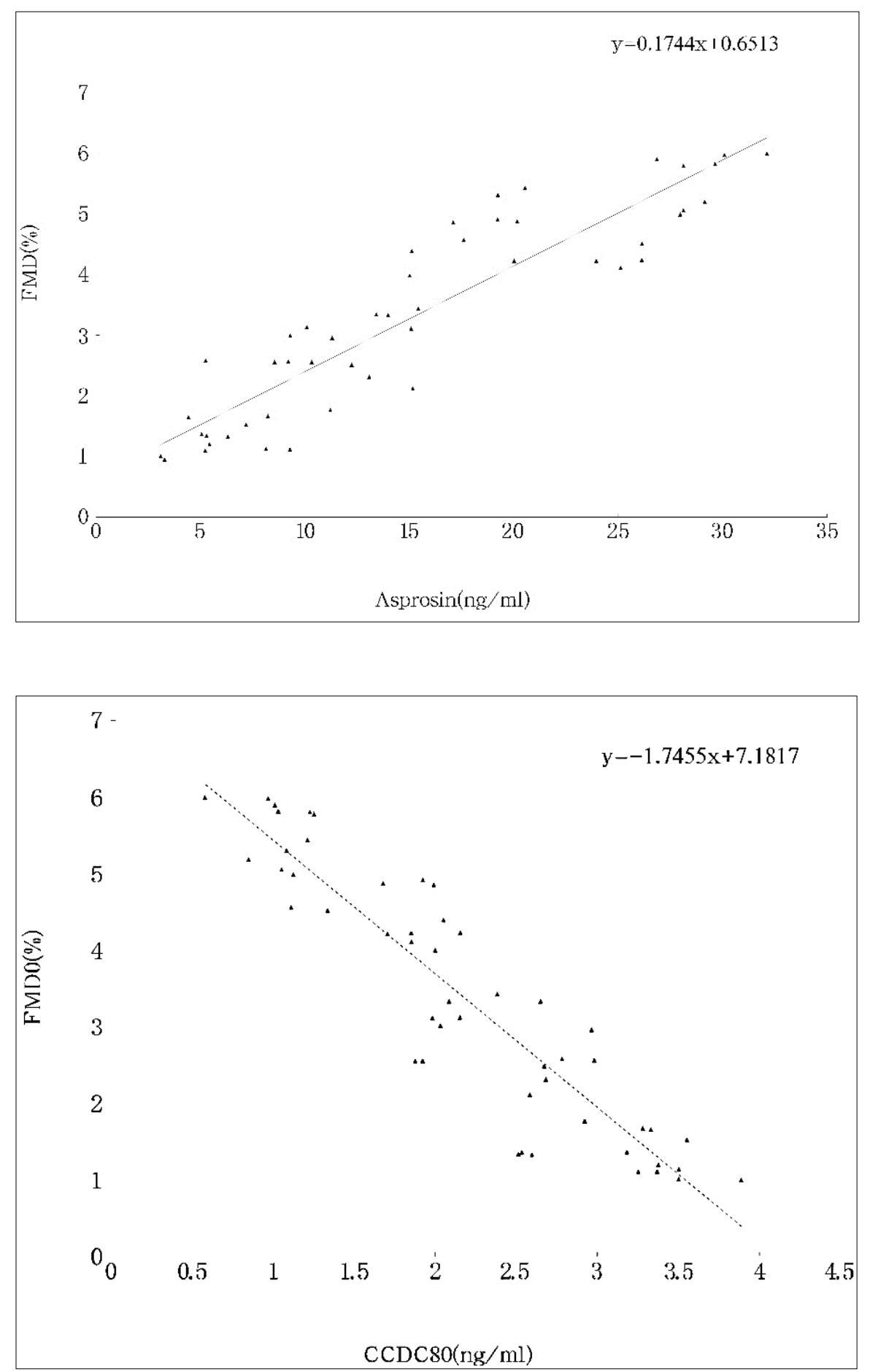

Fig. 1. Correlation between asprosin and FMD in patients with IBD
Fig. 2. Correlation between CCDC80 and FMD in patients with IBD 


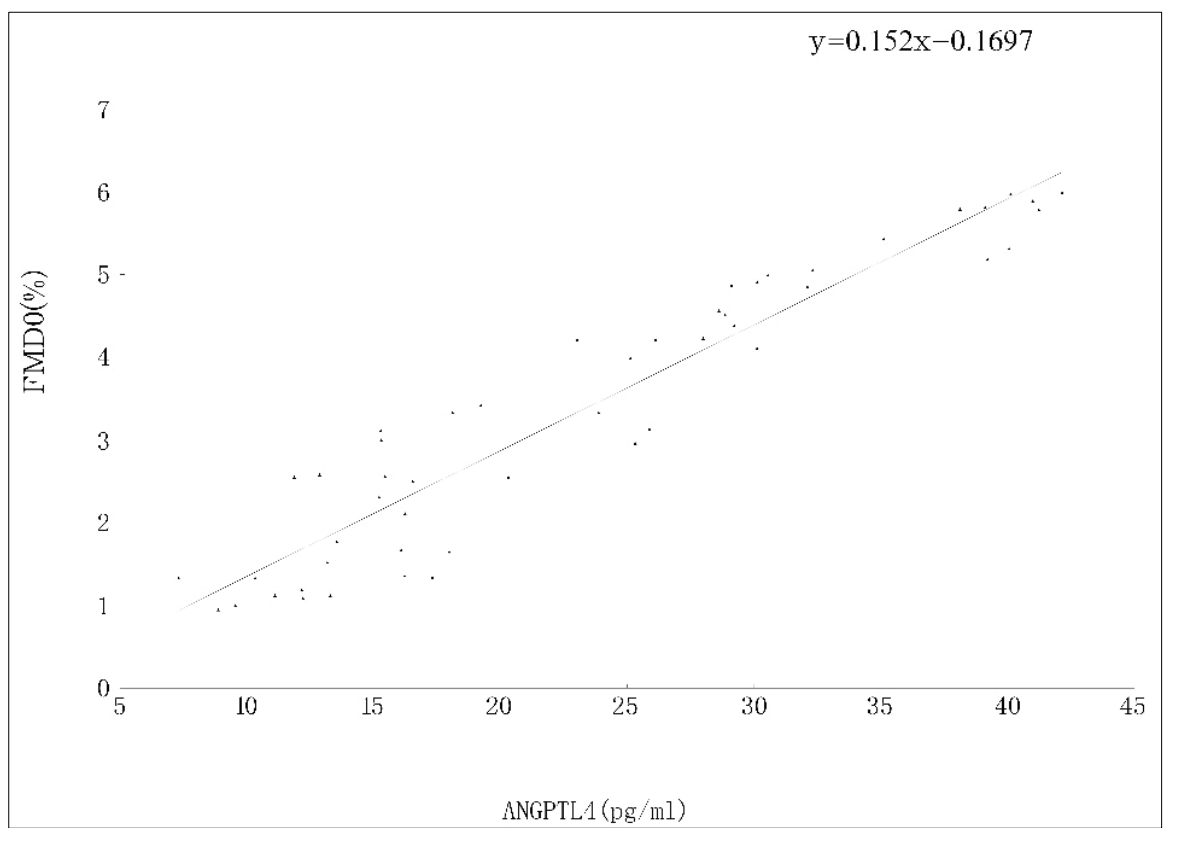

Fig. 3. Correlation berween ANGPTL4 and FMD in patients with IBD

\section{Discussion}

Adipocytokines regulate insulin secretion and insulin sensitivity, and play an important role in the pathogenesis of cardiovascular diseases. There are few studies on the plasma asprosin, CCDC80 and ANGPTL4 levels in IBD, nor on the relationship among the adipocytokines above, insulin resistance and endothelial function. Results of the present study have shown that plasma asprosin and ANGPTL4 levels are significantly decreased, and CCDC80 levels are significantly increased in both $\mathrm{CD}$ and $\mathrm{UC}$, especially in $\mathrm{CD}$. In addition, there were significant differences between IBD and bacterial or viral bowel inflammation for plasma asprosin, CCDC80 and ANGPTL4 levels. Moreover, asprosin, CCDC80 and ANGPTL4 were associated with insulin resistance and endothelial function in both $\mathrm{CD}$ and UC. Our findings strongly indicate that plasma asprosin, CCDC80 and ANGPTL4 levels may be applied as a significant marker for early stage of insulin resistance and atherosclerosis in patients with in IBD.

Insulin resistance contributes to the pathogenesis of type 2 diabetes and is closely linked with cardiovascular risk factors. Recently, it is well documented that IBD is often associated with insulin resistance ( $\mathrm{Ng}$ et al. 2018, Cibor et al. 2016). Our data have shown that insulin resistance represented by HOMA-IR was higher in CD or UC as compared to control-a and control-b. Some studies have shown the role of the chronic low-grade inflammatory response in the pathogenesis of insulin resistance (Matulewicz et al.
2016). The inflammatory process of IBD may also directly increase the risk of insulin resistance. Besides, patients with IBD have a greater prevalence of vitamin D deficiency than the healthy population (Seyed et al. 2019). Vitamin D deficiency contributes to both the initial insulin resistance and the subsequent onset of diabetes caused by $\beta$-cell death (Berridge 2017). Therefore, IBD is associated with insulin resistance and the mechanism needs to be further studied.

Plasma asprosin, CCDC80 and ANGPTL4 play important roles in the occurrence of insulin resistance (McCulloch et al. 2020, Min et al. 2019, O OsorioConles et al. 2017, Shota et al. 2016). The crucial mechanism of the relationship between IBD and insulin resistance remain unclear. In the present study, we measured plasma asprosin, CCDC80 and ANGPTL4 levels in both $\mathrm{CD}$ and $\mathrm{UC}$, and found that standard treatment change the levels of plasma asprosin, CCDC80 and ANGPTL4. Correlation analysis showed that the change in HOMA-IR in patients over the study period was correlated with the changes in asprosin, CCDC80 and ANGPTL4. It is well established that oxidative stress plays a major role in insulin resistance (Onyango 2018). Additionally, much evidence supports a pivotal role for ANGPTL4, CCDC80 and asprosin in oxidative stress (Anastasia et al. 2020, Zhengbin et al. 2019, De Lima et al. 2020). Stimulation of cardiac ANGPTL4 gene expression protected the heart against fatty acid-induced oxidative stress (Anastasia et al. 2020). Asprosin protects mesenchymal stromal cells from oxidative stress-induced apoptosis (Zhengbin et al. 2019). Gene expression 
of CCDC80 was associated with multiple feed efficiencyrelated traits and were previously related to glucose homeostasis, oxidative stress, fat mass, and osteoblastogenesis (De Lima et al. 2020). Accumulating evidence shows that oxidative stress plays an essential role in the pathogenesis and progression of IBD (Kruidenier et al. 2003, Goyette et al. 2007). Kruidenier, et al have showed that the markers of oxidative stress were dramatically upregulated in IBD (Kruidenier et al. 2003). Oxidative stress leads to damages of the mucosal layer in the gastrointestinal tract and bacterial invasion, which in turn stimulates the immune response and initiates IBD (Goyette et al. 2007). Of note, we hypothesized that the up regulation of CCDC80 induces the secretion of oxidative stress, and decrease the expression of asprosin and ANGPTL4, which may in turn result in insulin resistance and inflammatory response. In addition, whether asprosin, ANGPTL4 and CCDC80 can affect each other, whether these cytokines can act as independent factors affecting insulin resistance, and whether there are complex regulatory networks between various cytokines with different functions, thus indirectly affecting insulin resistance, still need to be confirmed by further research.

IBD is associated with an increased risk for cardiovascular disease and diminished FMD exists in IBD ( $\mathrm{Ng}$ et al. 2018, Cibor et al. 2016). The present results showed that FMD levels decresed in both $\mathrm{CD}$ and UC, compared to control-a and control-b, which is consistent with the previous studies. Our correlation analysis showed that FMD was correlated with CRP, ESR, ANGPTL4, CCDC80 and asprosin. We also found that the change in FMD in patients over the study period was correlated with the changes in asprosin, CCDC80 and ANGPTL4. The asprosin, CCDC80 and ANGPTL4 are involved in hyperglycemia, hypertension, obesity, abnormal lipid metabolism, and oxidative stress. These risk factors are all involved in the pathogenesis of atherosclerosis. In the present study, the alteration of asprosin, CCDC80 and ANGTPL4 could not well control blood sugar, blood pressure, obesity, lipid metabolism, and oxidative stress, inhibit inflammatory responses and protect the vascular endothelium. Additionally, nitric oxide (NO) is the main signal molecule that regulates vasodilation. There is a low NO availability in IBD that consequently a vasoconstriction occurs, because of smooth muscle cell relaxation reduction (Magro et al. 2014). Asprosin and ANGPTL4 are involved in NO production (Sha et al. 2019, Han et al. 2014).
Asprosin reduced reactive oxygen species production, reduced apoptosis and increased NO production in cardiac microvascular endothelial cells (Sha et al. 2019). ANGPTL4 stimulates signal transducer and activator of transcription3 mediated iNOS expression and enhances angiogenesis to accelerate wound healing in diabetic mice (Han et al. 2014). The levels of asprosin and ANGPTL4 in IBD, might inhibit NO synthesis, then stress the aorta and the resistance of blood vessels and destroy the blood vessel endothelium. Besides, CCDC80 down-regulated extracellular regulated protein kinase $1 / 2$ phosphorylation, which leaded to a reduction of lipoprotein lipase expression and acceleration of atherosclerosis (Duo et al. 2019). In short, asprosin, CCDC80 and ANGPTL4 levels in both $\mathrm{CD}$ and $\mathrm{BC}$ may exert its functions through different mechanisms of action.

Interestingly, we found that there were significant differences in plasma asprosin, CCDC80 and ANGPTL4 levels in CD when compared to UC. CD might cause severe systemic symptoms, such as fever, malnutrition and a larger range of disease. Compared with $\mathrm{CD}$, UC might locate in the limited distribution, such as sigmoid colon and rectum, which leads to moderate systematic reactions. Plasma asprosin, CCDC80 and ANGPTL4 levels might be used as indicators to differentiate $\mathrm{UC}$ from $\mathrm{CD}$, or to evaluate the prognosis. The present study has several limitations. Firstly, the enrollment population was limited, the number of samples was small, and the observation time was short. In later studies, the study population and sample size should be further expanded. Secondly, we speculate that asprosin, CCDC80 and ANGPTL4 might induce oxidative stress in patients with IBD. However, there is no comparative study on the effects of oxidative stress inhibitor and plasma asprosin, CCDC80 and ANGPTL4 levels in patients with IBD. Thirdly, the mechanism for the relationship among the adipocytokines above, NO and cell singling in patients with IBD unexplained completely. Further studies were needed to investigate the effect of plasma asprosin, CCDC80 and ANGPTL4 levels in patients with IBD.

\section{Conflict of Interest}

There is no conflict of interest.

\section{Acknowledgements}

The authors would like to thank Dr. Xiang Guang-da for excellent technical support and critically reviewing the manuscript. 


\section{References}

BERRIDGE MJ: Vitamin D deficiency and diabetes. Biochem J 474: 1321-1332, 2017. https://doi.org/10.1042/BCJ20170042

CELEMAJER DS, SORENSEN KE, GOOCH VM, SPIEGELHALTER DJ, MILLER OI, SULLIVAN ID, LIOYD JK, DEANFIELD JE: Non-invasive detection of endothelial dysfunction in children and adults at risk of atherosclerosis. Lancet 340: 1111-1115, 1992. https://doi.org/10.1016/0140-6736(92)93147-F

CIBOR D, DOMAGALA-RODACKA R, RODACKI T, JURCZYSZYN A, MACH T, OWCZAREK D: Endothelial dysfunction in inflammatory bowel diseases: Pathogenesis, assessment and implications. World J Gastroenterol 22: 1067-1077. 2016. https://doi.org/10.3748/wjg.v22.i3.1067

DE LIMA AO, KOLTES JE, DINIZ WJS, DE OLIVEIRA PSN, CESAR ASM, TIZIOTO PC, AFONSO J, DE SOUZA MM, PETRINI J, ROCHA MIP, CARDOSO TF, NETO AZ, COUTINHO LL, MOURÃO GB, REGITANO LCA: Potential biomarkers for feed efficiency-related traits in nelore cattle identified by co-expression network and integrative genomics analyses. Front Genet 11: 189, 2020. https://doi.org/10.3389/fgene.2020.00189

DUO G, QIANG Z, LING-YAN C, XIAO-HUA Y, GANG W, JIN Z, XI-LONG Z, DA-WEI Z, WEI-DONG Y, CHAO-KE T: Coiled-coil domain-containing 80 accelerates atherosclerosis development through decreasing lipoprotein lipase expression via ERK1/2 Phosphorylation and TET2 Expression. Eur J Pharmacol 843: 177-189, 2019. https://doi.org/10.1016/j.ejphar.2018.11.009

GEORGIADI A, LICHTENSTEIN L, DEGENHARDT T, BOEKSCHOTEN MV, VAN BILSEN M, DESVERGNE B, MULLER M, KERSTEN S: Induction of cardiac angptl4 by dietary fatty acids is mediated by peroxisome proliferator-activated receptor beta/delta and protects against fatty acid-induced oxidative stress. Circ Res 106: 1712-1721, 2010. https://doi.org/10.1161/CIRCRESAHA.110.217380

GOYETTE P, LABBE C, TRINH TT, XAVIER RJ, RIOUX JD: Molecular pathogenesis of inflammatory bowel disease: genotypes, phenotypes and personalized medicine. Ann Med 39: 177-199, 2007. https://doi.org/10.1080/07853890701197615

HAN CC, JEREMY SOON KC, CHI QG, NATALIA VG, BAIWEN L, XIAOLING W, SELIN F, MARCUS THIEN CW, CLEO C, SANDER K, NGUAN ST: Angiopoietin-like 4 stimulates STAT3-mediated iNOS xpression and enhances angiogenesis to accelerate wound healing in diabetic mice. Mol Ther 22: 1593-1604, 2014. https://doi.org/10.1038/mt.2014.102

HAO-HUA W, MIN L, GUANG-DA X: Serum HMGB1 Levels and its association with endothelial dysfunction in patients with polycystic ovary syndrome. Physiol Res 67: 911-919, 2018. https://doi.org/10.33549/physiolres.933831

JEHLICKA P, HUML M, SCHWARZ J, TREFIL L, KOBR J, SYKORA J: Reactive hyperaemia index as a marker of endothelial dysfunction in children with Crohn's disease is significantly lower than healthy controls. Acta Paediatr 103:e55-60.2014. https://doi.org/10.1111/apa.12467

KORKMAZ H, SAHIN F, IPEKCI SH, TEMEL T, KEBAPCILAR L: Increased pulse wave velocity and relationship with inflammation, insulin, and insulin resistance in inflammatory bowel disease. Eur J Gastroenterol Hepatol 26: 725-732, 2014. https://doi.org/10.1097/MEG.0000000000000104

KRUIDENIER L, KUIPER I, VAN DUIJIN W, MARKLUND SL, VAN HOGEZAND RA, LAMERS CB, VERSPAGET HW. Differential mucosal expression of three superoxide dismutase isoforms in inflammatory bowel disease. J Pathol 201: 7-16, 2003. https://doi.org/10.1002/path.1407

MAGRO F, SOARES JB, FEMANDES D: Venous thrombosis and prothrombotic factors in inflammatory bowel disease. World Journal of Gastroenterology 20: 4857-4872, 2014. https://doi.org/10.3748/wjg.v20.i17.4857

MATULEWICZ N, KARCZEWSKA-KUPCZEWSKA M: Insulin resistance and chronic inflammation. Postepy Hig Med Dosw (Online) 70: 1245-1258, 2016.

MCCULLOCH LJ, BRAMWELL LR, KNIGHT B, KOS K: Circulating and tissue specific transcription of angiopoietin-like protein 4 in human Type 2 diabetes. Metabolism 106: 154192, 2020. https://doi.org/10.1016/j.metabol.2020.154192 
MIN W, CHUNYAN Y, LING W, YUSHENG L, HONGGANG L, MIN L, XIAOQING Y, YANFENG X: Serum asprosin concentrations are increased and associated with insulin resistance in children with obesity. Ann Nutr Metab 5: 205-212, 2019. https://doi.org/10.1159/000503808

MORHARDT TL, WINTER HS: Measurement of microvascular function in pediatric inflammatory bowel disease. J Pediatr Gastroenterol Nutr 68: 662-668. 2019. https://doi.org/10.1097/MPG.0000000000002252

OSORIO-CONLES O, GUITART M, MORENO-NAVARRETE J M, EXCOTE X, DURAN X, FEMANDEZ-REAL J M, GOMEZ-FOIX A M, FEMANDEZ-VELEDO S, VENDRELL J: Adipose tissue and serum CCDC80 in obesity and its association with related metabolic disease. Mol Med 23: 225-234, 2017. https://doi.org/10.2119/molmed.2017.00067

ONYANGO AN: Cellular stresses and stress responses in the pathogenesis of insulin resistance. Oxid Med Cell Longev 2018: 4321714, 2018. https://doi.org/10.1155/2018/4321714

SHA C, XIONG W, CHEN-MING Q, JUAN-NI H, XIAO-YU W, CHAO-XUE X, MING-YANG T, RUI Z, HAIFENG P: Study of the role and mechanism of asprosin/spartin pathway in cardiac microvascular endothelial injury induced by diabete mellitus. Sichuan Da Xue Xue Bao Yi Xue Ban 50: 827-834, 2019.

SHOTA S, YUHEI N, HIROFUMI S, ERQUAN Z, SHIKO O, SOICHIRO M, YOSHIFUMI A, MIZUKI Y, KOKI K, REIKO K, YOSHIHIDE M, KAZUO M, TOSHIO T: Comparative transcriptome analysis identifies ccdc80 as a novel gene associated with pulmonary arterial hypertension. Front Pharmacol 7: 142, 2016. https://doi.org/10.3389/fphar.2016.00142

NG SC, SHI HY, HAMIDI N, UNDERWOOD FE, TANG W, BENCHIMOL EI, PANACCIONE R, GHOSH S, WU JCY, CHAN FKL, SUNG JJY, KAPLAN GG: Worldwide incidence and prevalence of inflammatory bowel disease in the 21st century: a systematic review of population-based studies. Lancet 390: 2769-2778, 2018.

SEYED MP, REZA JE, SEYED MH, ALI MOTTAGHI M, ANAHITA G, MARYAM G, MOHADESE AN, MAJID K, MAJID GM, GORDON AF, SEYED MRP, AMIR A: Vitamin D in inflammatory bowel disease: From biology to clinical implications. Complement Ther Med 47: 102189, 2019.

ZHENGBIN Z, YANZHEN T, LIWEN Z, BING Z, PAN F, ERHE G, CHENNIAN X, XIAOMING W, WEI Y, YANG S: Asprosin improves the survival of mesenchymal stromal cells in myocardial infarction by inhibiting apoptosis via the activated ERK1/2-SOD2 Pathway. Life Sci 231: 116554. 2019. https://doi.org/10.1016/j.1fs.2019.116554 